\title{
COMPORTAMENTO DAS VARIÁVEIS CARDIORRESPIRATÓRIAS DURANTE USO DO CICLOERGÔMETRO ATIVO NA UNIDADE DE TERAPIA INTENSIVA
}

\footnotetext{
Júlio César Nascimento Dantas*, Janmille de Sá Neves**, Pedro Henrique Cerqueira de Andrade**, Bruno Prata Martinez ${ }^{* * *}$, Jorge Luis Motta dos Anjos****

Autor correspondente: Bruno Prata Martinez - brunopmartinez@hotmail.com

* Fisioterapeuta do Hospital Cardiopulmonar e Hospital da Cidade.

** Fisioterapeuta Residente do Programa Multiprofissional em Saúde, Núcleo Unidade de Terapia Intensiva (UTI), pela Universidade do Estado da Bahia (UNEB).

*** Fisioterapeuta no Hospital Aliança, professor na Universidade do Estado da Bahia (UNEB)

**** Fisioterapeuta, Coordenador do Serviço de Fisioterapia do Hospital Geral Roberto Santos (HGRS).
}

\section{Resumo}

Objetivo: Avaliar o comportamento das variáveis cardiorrespiratórias durante o uso do cicloergômetro em membros superiores e inferiores em pacientes internados em uma unidade de terapia intensiva. Métodos: Trata-se de estudo retrospectivo onde os dados foram extraídos dos prontuários e ficha de coleta, em momentos diferentes e comparados entre si. As alterações fisiológicas agudas durante o uso do cicloergômetro foram coletadas durante a intervenção (5,15 e 30 minutos de atividade) e após. As variáveis avaliadas foram pressão arterial sistólica(PAS), diastólica(PAD) e média(PAM), frequência cardíaca (FC), duplo-produto(DP), saturação periférica de oxigênio $\left(\mathrm{SpO}_{2}\right)$, frequência respiratória( $(F R)$ e Borg. Essas mensurações foram realizadas conforme o tempo de tolerância do paciente a técnica. Resultados: A amostra foi composta por 29 pacientes, sendo que na análise comparativa entre as alterações no cicloergômetro realizado com MMII e MMSS, não foi observado diferença estatística entre as duas formas $(8,5 \pm 4,9$ vs $11,5 \pm 9,2$ minutos; valor de $p>0,05)$, bem como não teve diferença entre o tempo de tolerância entre a técnica realizada com MMII e MMSS $(8,5 \pm 4,9$ vs 11,5 $\pm 9,2$ minutos; valor de p: 0,281 ). A maioria dos pacientes tiveram como critério de interrupção a alteração do Borg $(91,2 \%)$, sendo que quase sempre associada a alteração de outras variáveis cardiorrespiratórias. Conclusão: O uso do cicloergômetro ativo para MMSS e MMII não apresentou diferença nas variáveis cardiorrespiratórias durante o uso em pacientes internados na UTI. Entretanto, o seu tempo de aplicação foi variável de acordo as respostas fisiológicas consideradas dentro dos valores de segurança. Palauras-chave: Fisioterapia; Unidades de Terapia Intensiva; Exercício. 


\title{
BEHAVIOR OF VARIABLE FOR ACTIVE CARDIORESPIRATORY CYCLE ERGOMETER USE IN INTENSIVE CARE UNIT
}

\begin{abstract}
Objective: To evaluate the cardiorespiratory variables when using the ergometer in the upper limbs and lower limbs in patients admitted to an intensive care unit. Methods: This is a retrospective study in which data were extracted from medical records and record collection, at different times and compared. Acute physiological changes while using the cycle ergometer were collected during the intervention (5,15 and 30 minutes of activity) and after. The variables were systolic blood pressure (SBP), diastolic blood pressure (DBP) and mean (MAP), heart rate (HR), double product (DP), oxygen saturation $\left(\mathrm{SPO}_{2}\right)$, respiratory rate (RR) and Borg. These measurements were performed according to the time technique patient tolerance. Results: The sample consisted of 29 patients, a comparative analysis of the changes in the cycle ergometer performed with lower limbs and upper limbs was no statistical difference between the two forms $(8.5 \pm 4.9$ vs $11.5 \pm 9.2$ minutes; value of $\mathrm{p}>0.05$ ) and had no difference between the tolerance time between the technique using the lower limbs and upper limbs $(8.5 \pm 4.9$ vs $11.5 \pm 9.2$ minutes; $p$-value: 0.281$)$. Most patients had to interrupt criterion changing the Borg (91.2\%), and almost always associated with changes in other cardiorespiratory variables. Conclusion: The use of active cycle ergometer for upper and lower limbs showed no difference in cardiorespiratory variables for use in ICU patients. However, its application time will vary according physiological responses considered within safe values.
\end{abstract}

Keywords: Physiotherapy; Intensive Care Unit; Exercise.

\section{INTRODUÇÃO}

Os pacientes criticamente enfermos que permanecem restritos ao leito por longos dias, comumente desenvolvem fraqueza muscular grave, causadas pela imobilidade, medicações e doenças, as quais acarretam sérios prejuízos aos sistemas cardiovascular, respiratório e neuromioarticular. ${ }^{(1)} \mathrm{Em}$ alguns casos os pacientes podem desenvolver neuromiopatia do doente crítico, devido ao uso de bloqueadores neuromusculares, sedativos e corticóides, os quais podem contribuir para um declínio de mobilidade, redução da sobrevida pós-alta, além de aumentar os custos hospitalares. ${ }^{(2,3)}$
Burtin et al. afirmam que a mobilização minimiza o impacto da imobilidade na funcionalidade e força muscular, quando instituído precocemente em pacientes internados nas unidades de terapia intensiva (UTI). ${ }^{(4)} \mathrm{Um}$ dos recursos terapêuticos descritas na literatura para isso é o cicloergômetro, além dos outros recursos como cinesioterapia (passiva, assistida, ativa livre e resistida), eletroestimulação funcional, treino de transferências, ortostatismo, treino de equílibrio e treino de marcha.

Estes podem ser usados na fase inicial da reabilitação física e funcional com o objetivo de restaurar a força muscular, ganho de amplitude de movi- 
mento, condicionamento cardiorrespiratório, além de auxiliarem para uma maior mobilidade no momento da alta. ${ }^{(2,4-6)} \mathrm{O}$ uso do cicloergômetro também já tinha sido citado pela força tarefa da European Respiratory Society and European Society of Intensive Care Medicine em 2008, como uma das atividades que podem contribuir para mobilização de pacientes na UTI e que pode ser iniciado de forma precoce, desde que o paciente apresente estabilidade cardiorrespiratória. ${ }^{(7)}$ Os autores afirmam que é preciso ter atenção com os fatores relacionados a segurança antes da execução da técnica, como aqueles intrínsecos ao paciente (histórico de doenças pregressa e atual, estabilidade clínica, reserva cardiorrespiratória) e fatores extrínsecos, como presença de cateteres, ambiente e equipe multiprofissional envolvida no processo. ${ }^{(8-10)}$

Sabendo-se dos benefícios potenciais do cicloergômetro para melhora do condicionamento cardiovascular, desempenho físico e dos possíveis riscos associados ao cicloergômetro em pacientes críticos, o presente estudo teve como objetivo avaliar o comportamento das variáveis cardiorrespiratórias durante o uso cicloergômetro ativo para membros superiores (MMSS) e membros inferiores (MMII) em pacientes internados numa UTI.

\section{MÉTODOS}

O presente trabalho foi um estudo retrospectivo sobre as alterações fisiológicas agudas durante o uso do cicloergômetro em membros superiores e membros inferiores dos pacientes que participaram do protocolo de cicloergômetro na UTI do hospital Geral Roberto Santos, Salvador-Bahia, no período de abril a maio de 2015 . Neste estudo, os dados foram extraídos dos prontuários e ficha de coleta, em momentos diferentes e comparados entre si. O estudo foi aprovado pelo Comitê de Ética e Pesquisa envolvendo seres humanos, respeitando todos os princípios éticos e a resolução 466/12, sob protocolo número 1.181.788.

Foram selecionados para o estudo os prontuários dos pacientes que foram internados na UTI da instituição durante o ano de 2014 e que fizeram parte do protocolo de exercício com a utilização do cicloergômetro da unidade, o qual consistia nos seguintes critérios: idade superior a 18 anos, capacidade de obedecer aos comandos, hemodinâmica estável (ausência de drogas vasoativas e/ou inotrópicas, pressão arterial sistólica entre 90 e $180 \mathrm{~mm}$ $\mathrm{Hg}$, frequência cardíaca entre 40 e $130 \mathrm{bpm}$ e ausência de doenças cardíacas agudas), (11) força muscular de MMSS e/ou MMII $\geq 3$ pelo teste de força muscular manual(12) e com relato verbal do paciente de independência funcional prévia a internação. A obtenção dos dados do prontuário se restringiu ao primeiro dia de realização do cicloergômetro, porém os pacientes continuavam realizando conforme estabilidade clínica e planejamento terapêutico. Foram excluídos todos os prontuários que não possuíam seus registros completos e com instabilidade hemodinâmica.

\section{PROTOCOLO PARA APLICAÇÃO DO CICLOERGÔMETRO}

A distribuição dos pacientes para realização do exercício no cicloergômetro ocorreu de acordo com uma avaliação prévia, que verificou a possibilidade da intervenção nos MMSS ou MMII. Se o paciente tivesse alguma restrição como por exemplo, acesso central periférico em MMSS, o mesmo era eleito para realizar o exercício em MMII. Caso não houvesse restrição para as duas regiões, a escolha era realizada de forma aleatória. Nenhum paciente realizou a técnica com os MMSS e MMII. A marca do cicloergômetro foi Liveup Sports, exercitador mecânico para membros superiores e inferiores, com monitor digital de tempo, distância, passos e calorias.

Após a distribuição dos pacientes no grupo de MMSS ou MMII e a identificação dos critérios de inclusão, os fisioterapeutas da unidade explicavam ao paciente sobre a atividade, orientando o mesmo a pedalar com um mesmo ritmo do início ao fim da atividade, de forma mais rápida possível. A posição para coleta de dados foi em sedestação com membros inferiores ou na cadeira para o cicloergôme- 
tro de MMII e no leito com cabeceira elevada para MMSS, sendo esclarecida pelo profissional a forma de realização da atividade. O tempo programado para a intervenção foi de no máximo 30 minutos, desde que os critérios de interrupção não fossem atingidos, sendo importante ressaltar que não foi realizado incremento de carga.

Como critérios de interrupção, foram utilizados a escala de percepção subjetiva de esforço do Borg Modificado (Borg) >7, angina, saturação periférica de oxigênio $\left(\mathrm{SpO}_{2}\right)<92 \%$, elevação da frequência cardíaca $(\mathrm{FC})>30 \%$ da encontrada em repouso, alterações na pressão arterial sistólica (PAS) e pressão arterial diastólica (PAD) superiores a $20 \%$ do basal, adaptados de Burtin. ${ }^{(13)}$ Os fisioterapeutas acompanharam durante todo tempo a realização do exercício para garantir a segurança da intervenção neste primeiro momento. A técnica foi interrompida antes dos 30 minutos quando ocorresse algum desses critérios anteriormente citados.

Durante o uso do cicloergômetro (aos 5, 15 e 30 minutos de execução da atividade, baseando-se em alguns estudos ${ }^{(7,10)}$ e após foram avaliados os valores de PAS, PAD, Pressão Arterial Média (PAM), FC, Duplo Produto (DP), $\mathrm{SpO}_{2}$, frequência respiratória (FR) e Borg, com um monitor da marca Dixtall (DX2O2O, Manaus-Amazonas, Brasil).

\section{ESTATÍSTICA}

A análise descritiva foi realizada através das médias e desvio-padrão e percentuais obtidos nas variáveis do estudo. Para comparação das alterações fisiológicas agudas durante os 5 minutos de uso do cicloergômetro foi utilizado o teste pareado $T$ de Student, sendo que para comparação entre a modalidade com MMII e MMSS foi utilizado o teste T de Student para amostras independentes.

\section{RESULTADOS}

Foram incluídos no estudo 29 pacientes com idade média de $48,0 \pm 18,1$ anos, sendo que a amostra foi composta por $15(51,7 \%)$ pacientes do sexo masculino. A maior parte dos pacientes tinha como motivo da internação problemas clínicos $(58,6 \%)$, sendo que no perfil cirúrgico todos foram pacientes no pós-operatório de cirurgia abdominal (41,4\%). O tempo médio de realização do cicloergômetro foi $9,9 \pm 7,3$ minutos, sendo que a frequência de uso em membros inferiores e superiores foram semeIhantes (tabela 1). Todos os 29 pacientes estavam em ventilação espontânea, sendo que destes, seis desses estavam sob uso de suporte de oxigênio. Nenhum paciente estava em uso de medicação beta-bloqueadora.

Tabela 1 - Dados descritivos da amostra de pacientes avaliados na UTI, Salvador-Bahia(n:29)

(continua)

\begin{tabular}{|c|c|c|}
\hline & MÉDIA $\pm \mathrm{DP}$ & $\mathrm{N} / \%$ \\
\hline Idade & $48, O \pm 18,1$ & \\
\hline \multicolumn{3}{|l|}{ Gênero } \\
\hline Masculino & & $15(51,7)$ \\
\hline \multicolumn{3}{|l|}{ Perfil admissional } \\
\hline Clínico & & $17(58,6)$ \\
\hline Cirúrgico & & $12(41,4)$ \\
\hline \multicolumn{3}{|l|}{ Motivo da internação } \\
\hline Pós-operatório abdominal & & $12(41,4)$ \\
\hline Sepse & & $10(34,5)$ \\
\hline Problemas cardiovasculares & & $4(13,8)$ \\
\hline Diabetes mellitus descompensada & & $2(6,9)$ \\
\hline Doença crônica parenquimatosa do fígado & & $1(3,4)$ \\
\hline
\end{tabular}


Tabela 1 - Dados descritivos da amostra de pacientes avaliados na UTI, Salvador-Bahia(n:29)

\begin{tabular}{lcc}
\hline & MÉDIA \pm DP & N / \% \\
\hline Modalidade do cicloergômetro & & $15(51,7)$ \\
MMII & & $14(48,3)$ \\
MMSS & $9,9 \pm 7,3$ & \\
Tempo da intervenção (minutos) & $8,5 \pm 4,9$ & \\
MMII & $11,5 \pm 9,2$ & \\
MMSS &
\end{tabular}

MMII: Membros inferiores; MMSS: membros superiores.

Todos os 29 pacientes que participaram do estudo conseguiram realizar a atividade do cicloergômetro por 5 minutos. Destes, somente dez conseguiram realizar a atividade até os 15 minutos, sendo que apenas dois completaram todo o exercício (30 minutos). Dentre os motivos para interrupção da técnica, 25 pacientes tiveram alterações dos parâmetros fisiológicos durante a realização da técnica e outros dois tiveram quadros álgicos em região abdominal após o início da técnica. A maioria dos pacientes tiveram como critério de interrupção a alteração do Borg $(91,2 \%)$, sendo que quase sempre associado a alteração de outras variáveis cardiorrespiratórias como pressão arterial, frequência cardíaca e respiratória. Após interrupção da técnica, houve melhora das alterações, sem relatos de ocorrência de eventos adversos associados de maior gravidade.
$\mathrm{Na}$ análise das alterações fisiológicas antes e após os 5 minutos do início do cicloergômetro, apenas a PAD não teve diferença significativa entre os dois momentos. De uma forma geral, houve elevação significativa das variáveis $F R, F C, P A S, P A M$, $\mathrm{DP}$ e Borg, com queda na $\mathrm{SpO}_{2}$, vide tabela 2. Após a realização do cicloergômetro, todos as variáveis cardiorrespiratórias retornaram ao seu estado basal antes do início da técnica. $\mathrm{Na}$ análise comparativa entre as alterações no cicloergômetro realizado com MMII e MMSS não foi observado diferença estatística entre as duas formas (tabela 3), bem como não teve diferença entre o tempo de tolerância entre a técnica realizada com MMII e MMSS $(8,5 \pm 4,9$ vs $11,5 \pm 9,2$ minutos; valor de p: 0,281$)$.

Tabela 2 - Comportamento das variáveis cardiorrespiratórias durante os 5 minutos de atividade no cicloergômetro, Salvador-Bahia(n:29)

\begin{tabular}{ccccc}
\hline & To* $^{*}$ & TI $^{*}$ & $\Delta$ BASAL-5MIN* & VALOR-P \\
\hline FR & $20,7 \pm 3,3$ & $30,1 \pm 5,9$ & $-9,4 \pm 5,8$ & 0,001 \\
FC & $92,2 \pm 15,2$ & $108,4 \pm 21,2$ & $-16,2 \pm 13,9$ & 0,001 \\
PAS & $123,0 \pm 18,1$ & $131,8 \pm 22,8$ & $-8,8 \pm 16,8$ & 0,009 \\
PAD & $71,8 \pm 14,1$ & $77,0 \pm 20,4$ & $-5,3 \pm 22,3$ & 0,214 \\
PAM & $89,1 \pm 14,2$ & $97,0 \pm 19,0$ & $-7,9 \pm 16,8$ & 0,017 \\
DP & $11,485 \pm 2,725$ & $14,412 \pm 4,067$ & $2,927 \pm 2,676$ & 0,001 \\
SpO2 & $96,8 \pm 1,6$ & $94,9 \pm 3,4$ & $1,9 \pm 3,1$ & 0,002 \\
Borg & $0,0 \pm 0,0$ & $6,4 \pm 3,8$ & $-6,4 \pm 3,8$ & 0,001 \\
\hline
\end{tabular}

FR: frequência respiratória; FC: frequência cardíaca; PAS: pressão arterial sistólica; PAD: pressão arterial diastólica; PAM: pressão arterial média; DP: duplo-produto; $\mathrm{SpO}_{2}$ : saturação periférica de oxigênio; To: momento basal/repouso; $\Delta$ basal-5min: delta das variáveis no momento inicial/basal para o final de 5 minutos de cicloergômetro; *: média/desvio-padrão. 
Tabela 3 - Comparação das médias das diferenças entre as variáveis cardiorrespiratórias avaliadas com o cicloergômetro para MMSS e MMII, Salvador-Bahia(n:29)

\begin{tabular}{cccc}
\hline & MMII & MMSS & Valor-p \\
\hline FR & $7,1 \pm 6,0$ & $7,9 \pm 4,1$ & 0,68 \\
FC & $15,5 \pm 11,1$ & $13,1 \pm 16,2$ & 0,64 \\
PAS & $11,7 \pm 18,9$ & $5,0 \pm 13,1$ & 0,27 \\
PAD & $7,2 \pm 14,8$ & $5,9 \pm 20,1$ & 0,85 \\
PAM & $9,3 \pm 17,0$ & $6,4 \pm 17,2$ & 0,64 \\
DP & $3,165 \pm 2,901$ & $2,152 \pm 2,525$ & 0,327 \\
SpO2 & $-1,5 \pm 2,2$ & $-2,6 \pm 3,8$ & 0,316 \\
Borg & $6,2 \pm 3,7$ & $7,2 \pm 3,6$ & 0,455 \\
\hline
\end{tabular}

FR: frequência respiratória; FC: frequência cardíaca; PAS: pressão arterial sistólica; PAD: pressão arterial diastólica; PAM: pressão arterial média; DP: duplo-produto; $\mathrm{SpO}_{2}$ : saturação periférica de oxigênio.

\section{DISCUSSÃO}

O presente estudo demonstrou que os pacientes submetidos ao exercício com cicloergômetro ativo tiveram elevação das variáveis cardiorrespiratórias, com melhora após interrupção do exercício. Apesar de alguns pacientes não conseguirem concluir o tempo total pela ocorrência de critérios de interrupção, não houveram relatos de intercorrências após a interrupção, o que demonstra que provavelmente não houve ocorrência de danos na amostra estudada. Stiller et al relataram que para garantir uma maior segurança ao paciente durante o cuidado prestado pela fisioterapia, a mobilização precoce em doentes críticos deve ser adequada, de modo que a intensidade, duração e frequência da intervenção respeitem a tolerância da reserva cardíaca e respiratória do paciente crítico. A monitorização antes, durante e após o exercício é essencial e recomenda-se a avaliação do padrão ventilatório, dos impactos expressivos na FC, PA, $\mathrm{SpO}_{2}$ e arritmias no eletrocardiograma. (9) Este estudo comparou os efeitos fisiológicos agudos do uso do cicloergômetro de MMSS e MMII através das variáveis supracitadas e demonstrou que é possível realizar o cicloergômetro de forma ativa com segurança.
No presente estudo, houve um aumento significativo das variáveis FC, PAS, PAM e manutenção da PAD, os quais podem ser justificados pelas alterações fisiológicas provenientes do exercício sobre o sistema cardiovascular como aumento da atividade nervosa simpática e diminuição da parassimpática, que promovem aumento da FC e volume sistólico e por consequência. ${ }^{(14,15)}$ Ainda sobre esse sistema, no exercício aeróbico como o cicloergômetro por exemplo, há vasodilatação da musculatura em atividade pela liberação de óxido nítrico, a qual resulta em redução da resistência vascular periférica. ${ }^{(14,15)} \mathrm{Em}$ virtude dessas alterações, a PAS se eleva e a PAD tende a se manter ou reduzir conforme ocorrido neste estudo.

Pires-Neto ${ }^{(8)}$ realizaram um estudo com o objetivo de analisar as alterações cardiorrespiratórias de pacientes durante o exercício ativo com um cicloergômetro para MMII por 5 minutos e verificaram um aumento significativo da FC, FR e Borg, as quais foram semelhantes ao encontrado neste estudo, $O$ que é esperado de forma fisiológica pelo aumento do consumo de oxigênio dos tecidos musculares e do sistema cardiorrespiratório. Importante ressaltar que no estudo citado, a maioria dos pacientes tinha o perfil clínico, semelhante a este estudo. No presente estudo, houve queda de forma estatistica- 
mente significante apenas para $\mathrm{SpO}_{2}$, porém dentro de limites considerados seguros.

Em relação as alterações cardiorrespiratórias entre os MMII e MMSS no presente estudo, não houve diferença do ponto de vista estatístico, apesar da PAS ter tido uma tendência a maior elevação no grupo de MMII. Não existiu diferença estatística também entre o tempo de tolerância entre as duas modalidades, apesar dos valores absolutos da técnica com MMSS ter tido um tempo superior a com os MMII.

Cordeiro $^{(16)}$ ao analisarem as alterações hemodinâmicas do treino com cicloergômetro em pacientes no pós operatório de cirurgia cardíaca observaram que apenas a FR apresentou variação e concluíram que a utilização do cicloergômetro no pós operatório de cirurgia cardíaca é uma prática segura. Apesar do perfil diferente de pacientes em relação ao nosso estudo, estes dados corroboram como o presente estudo e alertam para uma maior prescrição do cicloergômetro no ambiente hospitalar, já que sabe-se que a mobilização precoce pode promover diversos benefícios para os pacientes críticos internados. ${ }^{(17-21)} \mathrm{O}$ estudo de Pires-Neto et al evidenciou que o uso de cicloergômetro em ambiente de terapia intensiva também pode trazer satisfação aos pacientes, sendo que todos eles gostariam de repetir a atividade na próxima sessão de fisioterapia. ${ }^{(8)}$ Além dessa adesão por parte dos pacientes, houve pequenas alterações cardiorrespiratórias, corroborando com os dados evidenciados nesta pesquisa.

Este estudo tem como limitação o pequeno número de pacientes, a ausência de uma escala de gravidade e tempo de internação dos pacientes, o que impossibilita a identificação dos pacientes com maior grau de complexidade. Não houve controle do ritmo desempenhado pelo paciente, apesar da orientação de manter a mesma frequência de pedalada até o final do exercício. Novos estudos sobre a utilização do cicloergômetro e sua relação com as repercussões hemodinâmicas e desfechos funcionais dos pacientes hospitalizados ainda são necessários.

\section{CONCLUSÃO}

O uso do cicloergômetro ativo para MMSS e MMII não apresentou diferença nas variáveis cardiorrespiratórias durante o uso em pacientes internados na UTI. Entretanto, o seu tempo de aplicação foi variável de acordo as respostas fisiológicas consideradas dentro dos valores de segurança.

\section{REFERÊNCIAS}

1. Brower RG. Consequences of bed rest. Crit Care Med. 2009;37(10 Suppl):S422-8.

2. Korupolu R, Gifford JM, Needham DM. Early Mobilization of Critically III Patients: Reducing Neuromuscular Complications After Intensive Care. Contemp Critical Care. 2009;6(9):1-12.

3. Vincent $\mathrm{JL}$, Norrenberg M. Intensive care unitacquired weakness: framing the topic. Crit Care Med. 2009; 37(10 Suppl): S296-8.

4. Burtin C, Clerckx B, Robbeets C, Ferdinande P, Langer D, Troosters T et al. Early exercise in critically ill patients enhances shortterm functional recovery. Crit Care Med. 2009;37(9):2499-505.

5. Needham DM. Mobilizing patients in the intensive care unit: improving neuromuscular weakness and physical function. JAMA. 2008;300(14):1685-1690.

6. Bourdin G, Barbier J, Burle JF, Durante G, Passant S, Vincent B, Badet $M$ et al. The feasibility of early physical activity in intensive care unit patients: A prospective observational one-center study. Respir Care. 2010;55(4):400-7.

7. Gooselink R, Bott J, Johnson M, Dean E, Nava $\mathrm{S}$, Norrenberg $\mathrm{M}$ et al. Physioterapy for adult patients with critical illness: recommendations of the European Respiratory Society and European Society of Intensive Care Medicine Task Force on Physioterapy for Critically ill Patients. Intensive Care Med. 2008;34(7):1188-99.

8. Pires-Neto RC, Pereira AL, Parente C, Sant'Anna GN, Eposito DD, Kimura A et al. Caracterização do uso do cicloergômetro para auxiliar no atendimento fisioterapêutico em pacientes críticos. Rev Bras Ter Intensiva. 2013; 25(1): 39-43. 
9. Stiller K, Phillpis A, Lambert P. The safety of mobilisation and its effect on haemodynamic and respiratory status of intensive care patients. Physiother Theory Pract. 2004;20(3):175185.

1O. Kathleen M, Vollman RS. Progressive mobility in the critically ill. Crit Care Nurse. 2O10;3O(2Suppl):S 3-5.

11. Schweickert WD, Pohlman MC, Pohlman AS, Nigos C, Pawlik AJ, Esbrook CL et al. Early physical and occupational therapy in mechanically ventilated, critically ill patients: a randomised controlled trial. Lancet. 2009;30;373(9678):1874-82.

12. Bittner EA, Martyn JA, George E, Frontera WR, Eikermann M. Measurement of muscle strength in the intensive care unit. Cri Care Med. 2009;37(10 suppl):S321-30.

13. Burtin C, Clerckx B, Robbeets C, Ferdinande P, Langer D,Troosters T, et al. Early exercise in critically ill patients enhances shortter, functional recovery. Crit Care Med. 2009;37(9):1-7.

14. Neto TLB, Tebexreni AS, Tambeiro VL. Aplicações práticas da ergoespirometria no atleta. Rev Soc Cardiol. 2001;3(11):695-705.

15. Brum PC, Forjaz CLM, Tinucci T, Negrão CE. Adaptações agudas e crônicas do exercício físico no sistema cardiovascular. Rev Paul Educ Fís. 2004;18(1):21-31.
16. Cordeiro AL, Barbosa AFN, Leitão LP, Araújo PAS, Carvalho S. Efeitos Hemodinâmicos do treino em cicloergômetro em pacientes no pós operatório de cirurgia cardíaca. Rev DERC. 2014; 2O(3): 90-93.

17. Stiller K. Safety issues that should be considered when mobilizing critically ill patients. Crit Care Clin. 2007; 23(1): 35-53.

18. Mota CM, Silva VC. A segurança da mobilização precoce em pacientes críticos: uma revisão de literatura. Interfaces Científicas-Saúde e Ambiente. 2012;24(1):93-91.

19. Cordeiro AL, Silva AA, Santana MI, Carvalho S, Guimarães AR. Impacto hemodinâmico da deambulação nos pacientes submetidos a cirurgia cardíaca. Rev DERC. 2015;21(2):5457.

2O. PE, Goad A, Thompson C, Taylor K, Harry $B$, Passmore $L$ et al. Early intensive care unit mobility therapy in the treatment of acute respiratory failure. Crit Care Med. 2008; 36(8):2238-43.

21. Soares TR, Avena KM, Oliveira FM, Feijó LF, Mendes KMB, Filho SAS et al. Retirada do leito após a descontinuação da ventilação mecânica: há repercussão na mortalidade e no tempo de permanência na unidade de terapia intensiva? Rev Bras Ter Intensiva. 2010;22(1): 27-32. 